\title{
Risk of Chlamydia/ Gonorrhoea NAAT contamination from clinic surfaces - need for patient and staff awareness in self-swabbing and pooling areas
} Harriet Wallace, Sharon Daley, Michelle Loftus-Keeling, Janet Wilson Leeds Sexual Health

\section{Background/introduction}

A self versus clinician Chlamydia/gonorrhoea (CT/NG) NAAT swab trial, with pooling of self-taken samples, recruited in Leeds between January 2015-September 2016. In addition to a pooled sample (see below), all participants had one self and one clinician sample from pharynx and rectum, and females did a vulvovaginal test and males a first void urine.

\section{Pooling}

\section{Females:}

- self-taken pharyngeal swab dipped into NAAT solution and agitated for 10 seconds

- Self-taken rectal sample dipped into NAAT solution and agitated for 10 seconds

-vulvovaginal sample taken and left in solution

- one result given from lab

Males:

-self-taken pharyngeal swab dipped into urine NAAT transport solution and agitated 10 seconds - self-taken rectal swab taken and left in solution - first void urine pipetted to fill line by clinician -one result given from lab

There was concern that nucleic acid contamination of clinic surfaces could be a source of false-positive samples during the pooling process, as this has previously been shown in laboratory studies (1) and noted in self and clinician sampling (2). If this risk was highlighted locally we hoped to influence practice to reduce contamination.

\section{Aim(s)/objectives}

- To ascertain levels of environmental nucleic acid contamination within our clinic environments.

- To determine number of false positive pooled samples throughout study

\section{Methods}

Environmental samples of clinic rooms, sluices and toilets were performed and tested using Aptima Combo 2 testing for chlamydia and gonorrhoea.

Swabs were rubbed on desks, door handles and couches in examination rooms, door handles in toilets and shelves and trolleys in sluice areas where urine was pipetted.

If contamination was found, the areas were retested after a standard clean. Room use continued.

In November 2015, the clinic relocated from old hospital premises to a newly renovated site.

Results were disseminated to staff throughout to raise awareness. Posters in self-swab areas highlighted risk of contamination, importance of hand-washing and no surface contact for swabs.

\section{Results}

Environmental

Two clinic sites were sampled:

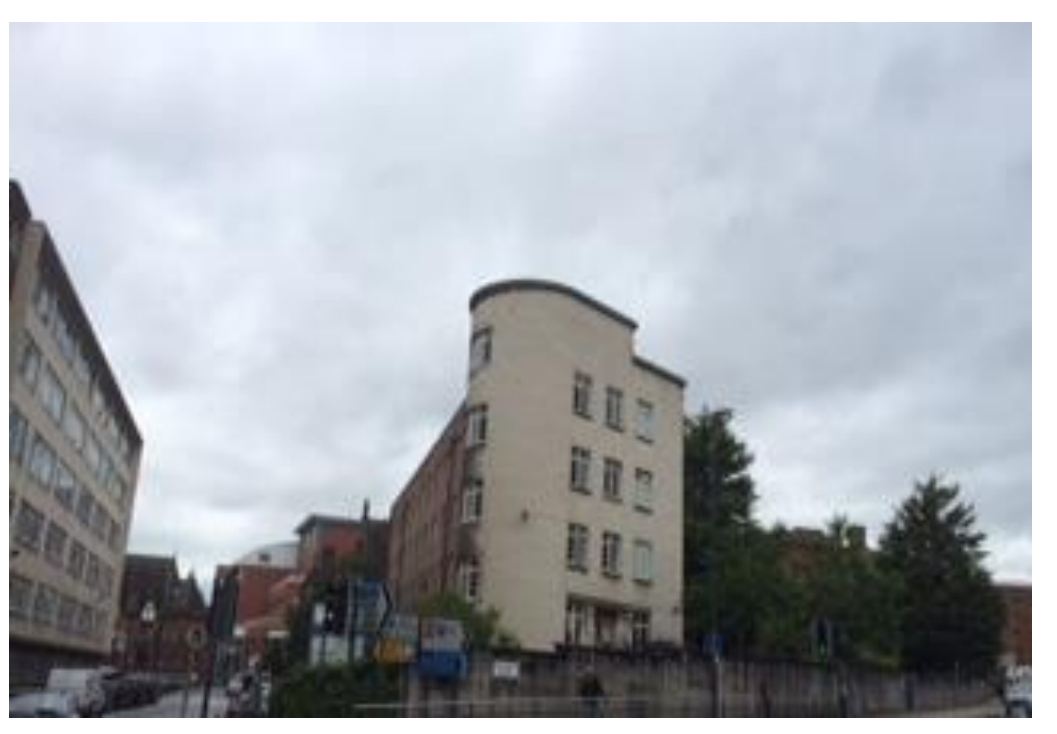

Site B

Merrion centre Balcony level, Leeds City centre

A purpose-adapted space with brand new rooms. Situated upstairs in a shopping centre, used from November 2015.

\section{Site A}

Sunnybank wing, Leeds General Infirmary

This longstanding site of a sexual health service in the city was last refurbished in 1995. Part of the old hospital site.

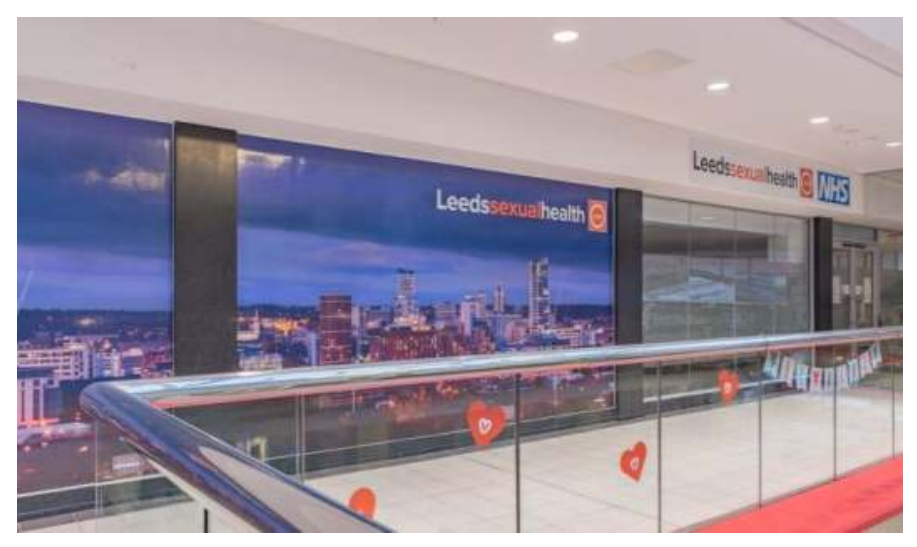

Of 41 environmental NAAT sampling episodes over a 12 month period within the study (March 2015-March 2016):

- $17(41 \%)$ were CT/GC positive/indeterminate.

- These were distributed throughout the whole 12 months.

- In Site A, 12/27 (44\%) samples were contaminated vs 5/14 (36\%) in site B

- Positive results were obtained from surfaces in all clinical examination rooms at site $A$, and toilets and sluices (where urines were pipetted) at both sites.

- 3 out of 4 clinic rooms regularly used for examination at the new site $B$ remained contamination free.

\section{Results}

False positive pooled samples:

Pooled samples were classed as false positive if all other CT/NG samples from that patient were negative.

There were 7 false positive pooled samples ( 6 female, 1 male); this was out of a total participant number of $1794(0.4 \%)$.

All false positives were in the first 8 months of the study.

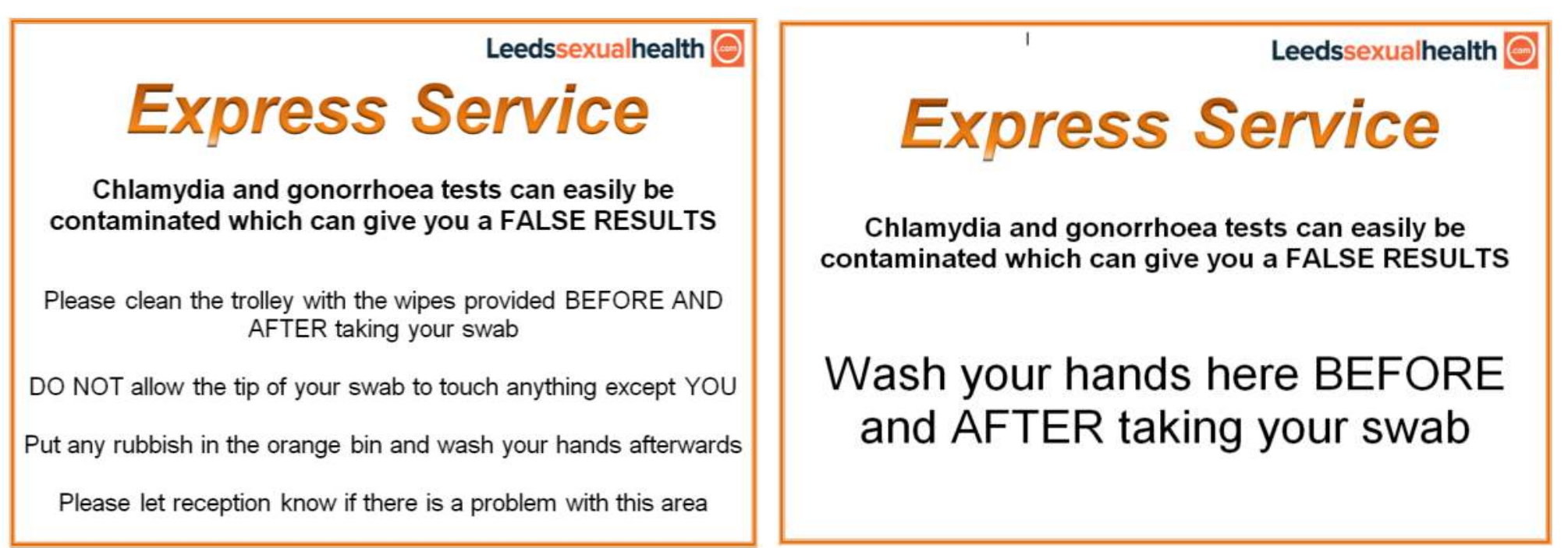

Examples of posters used in patient self-swab areas

\section{Conclusion / discussion}

It has previously been noted that environmental contamination is often low level and likely insufficient for transmission (3). However, a diagnosis of an STI when there is none for a patient can be distressing and impact on relationships, as well as resulting in unnecessary treatment.

Staff pipetting urine in this pooling study may have been a factor for a lower number of false positives in males. No false positives after the first 8 months suggests increased awareness had a positive effect on practice.

From the overall low rates of pooling contamination $(0.4 \%)$ we demonstrate that the impact of known clinic environment contamination can be minimised by raising awareness amongst staff and patients.

References:

1. Meader E et al. Chlamydia trachomatis RNA in the environment: is there potential for false-positive nucleic acid amplification test results? Sex Transm Infect 2008; 84(2):107-10

2. Lewis N et al. Chlamydia and gonorrhoea contamination of clinic surfaces. Sex Transm Infect 2012;88(6):418-21

3. Ross J. Nucelic acid contamination in sexual health clinics Curr Opin Infect Dis 2015;28(1): $80-2$ 